\title{
Meta-model for Data Harmonization and Business Process Compliance in Service Procurement
}

\author{
Maik Herfurth ${ }^{1}$, Thomas Schuster ${ }^{1}$, and Peter Weiß ${ }^{2}$ \\ ${ }^{1}$ FZI Forschungszentrum Informatik, Karlsruhe, Germany \\ ${ }^{2}$ ISS International School of Services, Hamburg, Germany \\ \{herfurth, schuster\} afzi.de \\ weiss@iss-hamburg.de
}

\begin{abstract}
Diminished transaction costs, increased transparency and shortened procurement times entail increased importance of service e-procurement compared to traditional service procurement. Due to proprietary data exchange formats and individual business processes, data harmonization and business process compliance are shortcoming. In this paper a meta-model to formulate requirements of service procurement collaborations with a combined view on information and control flow is presented. Our focus is on two constituent elements of the model: collaborative business processes and service objects. Both elements define features which are characteristic for investigated value constellations. Challenge at hand is to implement standardized interfaces easing seamless exchange of information between business partners. Existing procurement systems need enhanced process-awareness and compliance with servicedominant-logic. The meta-model also serves as reference framework which identifies and correlates typical entities of service procurement collaborations. Furthermore a domain specific meta-model extension for industrial service procurement is derived. This extension serves as basis for data harmonization of service procurement data and enacts compliance of business processes in industrial service procurement collaborations.
\end{abstract}

Keywords: Collaborative business processes, service procurement, business process compliance, data harmonization, meta-modeling, reference-modeling.

\section{Introduction}

Service procurement collaborations in service chain networks are gaining more attention since industrial services contribute progressively more to value creation of organizations. Industrial services present more than 40 percent of revenues in the industrial sector [13]. Especially in the sector of the machine and plant manufacturing, the relevance of e-business for services especially of service procurement is seen as a decisive factor for the long term success in a competitive environment [7]. Capital goods producer and service providers are organized locally. Therefore complex value chains evolve from industrial service chain network structures. The paper focuses on industrial services, especially product-related services. 


\section{Challenges of Service Procurement}

The collaboration of service providers and requesters poses challenges for the definition of business processes and data for service procurement [18]. An integrated perspective of goods and services is in focus of industrial service procurement [1]. Industrial service procurement is source of high cost because underlying business processes are error prone in most companies. Errors and failures occur foremost at the interface between buyer and supplier. This can be explained through the absence of coherent e-business standards and reference frameworks offering meta-models of processes, data objects and interaction patterns. Although, a variety of e-business standards and frameworks is available, they typically cover solely parts and phases of business processes, and causing numerous media breaks as well as require manual error and exception handling. Service procurement meets challenges based on the characteristic attributes of services [16]. These characteristics influence business processes and data structures. Business processes and data structures for service procurement collaborations aren't harmonized yet. Harmonization is defined as combined term of integration, standardization, consolidation, synchronization and coordination [15]. Business processes must support interaction and communication between service suppliers and requesters [12] [10]. Process compliance addresses the need of harmonized business processes in order to ensure and facilitate compliance with regulations. For the harmonization of business processes, different approaches like process integration [3], process orientation [5], process patterns [6] or reference process models [4] can be utilized [14]. Data harmonization enables integration of information systems for service procurement orders. For data harmonization identical objects with different syntax must be adapted. Data structures must support complex industrial services descriptions as well as service transaction document types [9] [10]. Therefore the creation of new collaborations hampers the business relations by increasing integration and transaction costs, offline communication and procurement times; also resulting in less transparency and low quality of processes and data [8]. Information systems have to be aware of business processes and related document flows. Systems that execute business processes control relevant tasks as well as coordinate complex flow of information and documents between organizations [11].

\section{$3 \quad$ Meta-model for Service Procurement}

In order to improve harmonization of business processes and data structures for the design and development of information systems in service procurement, a MOFcompliant meta-model is developed. This meta-model defines requirements for business processes and data structures of service chain collaborations. Relevant entities are associated with each other. The meta-model serves as a conceptual model for developing further concrete models such as a reference of process object models. This information is gathered in the meta-models. The meta-model documents elicited requirements concerning types of business interactions in the domain of industrial services. It is focused on four perspectives: (1) collaboration, (2) organization and 
resources, (3) business processes and (4) data. As information model, the meta-model enables the definition and evaluation of requirements and serves as a basis for development of information systems. The modeling is motivated by the design of business processes and data structures. Due to a harmonization of data exchange, the collaboration of service suppliers and service requesters can be improved by the development of common or new e-business standards. The meta-model is presented with Unified Modeling Language (UML) class diagrams.

\subsection{Requirements}

For the development of the meta-model, general requirements are formulated.

- Formal and graphical representation: To apply a precise and formal modeling notation to represent relevant entities.

- Consistency: To provide a consistent composition to avoid redundancies. A consistent modeling approach improves the perpetuity of model elements.

- Distribution and independence: Collaborative use and distribution for different involved parties. Use of a neutral representation and graphical notation ensures further development for software services and applications.

- Principles of proper modeling: Principles of proper modeling [2] encompass design recommendations concerning syntactic and semantic correctness, relevance, economic efficiency, clearness and comparability.

Furthermore context specific requirements are the refinement of choreography and collaboration aspects. The choreography defines a certain perspective of collaboration and describes the interaction of the two collaboration parties via document- and/or message-oriented inter-organizational processes. Thus relevant interrelations between entities in service chain collaborations should be described.

\subsection{Model Perspectives}

The meta-model is described by different model perspectives: collaboration model, organization and resources model, data model and business process model. The collaboration and organizational and resource model perspective are presented below.

Collaboration Model Perspective. The class CollaborativeBusinessProcess defines instances of collaborative business processes. ServiceChainProcess is a specialization of CollaborativeBusinessProcess and possesses additional attributes representing the specificities of service processes. Bilateral and multilateral collaborations can be differentiated. The class ChoreographyActivity represents choreography activities. The choreography defines the order of collaborative activities by message exchanges. The classes ReceiveActivity and SendActivity are sub classes of ProcessActivity for sending and receiving messages. The class LegalTerms defines contractual agreements of collaborations. 
Organization and Resource Model Perspective. For tasks and business processes, roles are defined and assigned to collaboration participants. The class Role defines role concepts and builds a super class of CollaborationParticipantRole. The class CollaborationParticipantRole defines roles participating in a collaboration. The class OrganizationalUnit defines organization units. A role of the class CollaborationParticipantRole is assigned to an instance of the class CollaborationParticipant. The super class Role as well as the sub class OrganizationUnit and colalborationParticipantRole are connected with the sub class HumanResource. Each role is completed by a human resource. Further subclasses of resource are information systems or organizations. The complete meta-model is shown in Fig. 1.

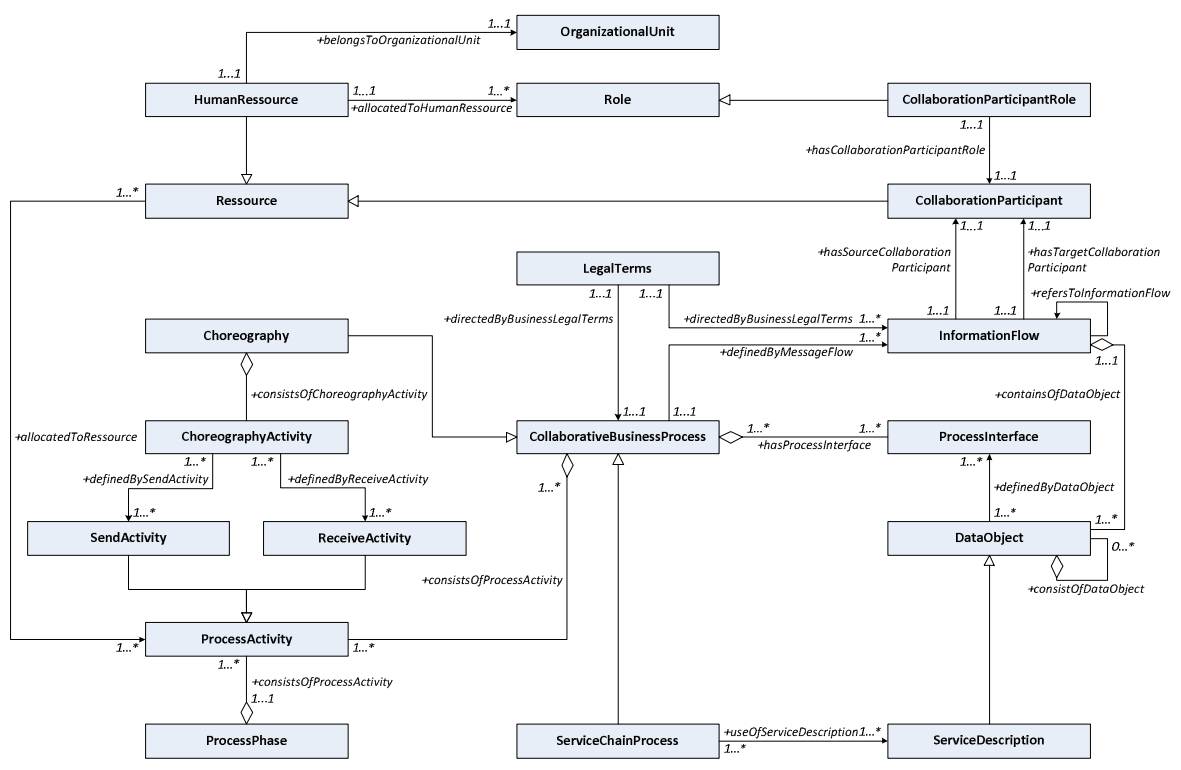

Fig. 1. Meta-model

\section{Meta-model Extension for Industrial Service Procurement}

For the support of information system design, the meta-model is extended concerning for requirements of bilateral industrial service procurement collaborations. Based on the meta-model extensions, domain specific models can be derived. The extensions of the meta-model are also additionally described by the model perspectives outlined above. 


\subsection{Requirements for the Meta-model Extension}

Context specific requirements for the meta-model extensions are focus on the data model and process model perspective:

- Data model perspective (service design): The design of service descriptions should consider specificities and properties of services. For the flexible design of service descriptions, modularization, standardization and variant definitions should be possible. Main factors of industrial services (resources) need to be described including the external factor of a service. Specifically for industrial services, the interaction place must be included in the service description. Electronic classification of services supports the grouping and coherent description offering a standardized coding system to be referenced by all business partners.

- Process model perspective (process structures and modularization): Process modularization and structuring improves the understanding of collaborative business processes and reduces complexity. Business processes can be subdivided into partial processes and allow for the building variants for business process models.

\subsection{Model Perspectives}

Extensions of the data model and process model perspectives are outlined below.

Data Model. The classes Dataobject and ServiceDescription are extended by adding specializations of these classes. The class BusinessTransactionDocument defines an abstract super class of specific transaction document types for service procurement (Fig. 2).

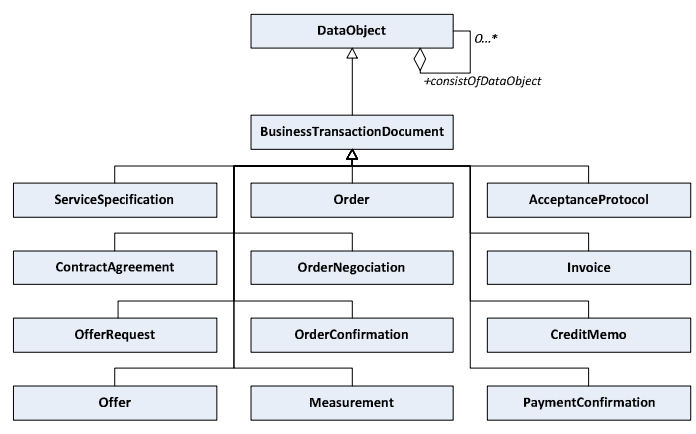

Fig. 2. Extensions of Dataobjects

The class ServiceDescription is extended by specific specialization of classes for the description of industrial services (Fig. 3). An industrial service description is a composite construct of ServicePosition, MaterialPosition and MachinePosition. The Relatedobject describes the external factor and is a sub class of MachinePosition. All these classes are connected with the class Classification. The class Classification assigns codes to groups of industrial services with 
similar attributes using a classification scheme (ClassificationScheme) and features (Features).

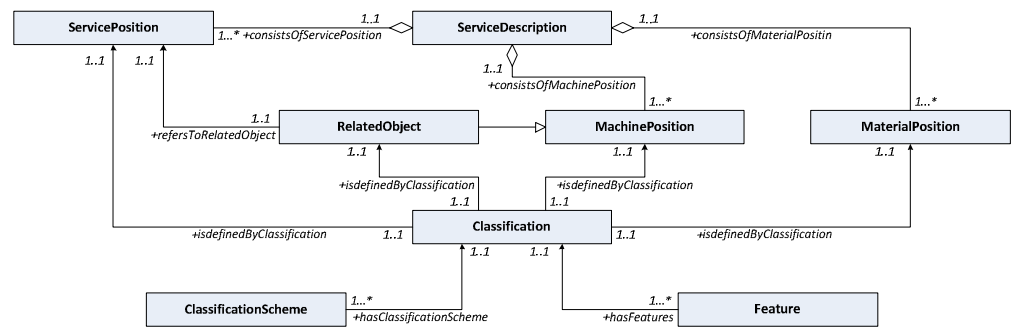

Fig. 3. Extension of ServiceDescription

Process Model. The classes CollaborativeBusinessprocess and ProcessPhase are associated with specialization classes possessing additional attributes and special behavior. A collaborative business process can be capsulated by process modules. A capsulated process of a process module is executed by one collaboration partner. Process modules are defined by the class CollaborativeProcessModule. Service procurement processes consist of different process phases. Business processes are assigned to one or more of respective process phases. The class Process Phase defines specific process phases of service procurement. The extended process phases are Predefinition, Request, Offer, Order, Execution, Measurement, Acceptance and Accounting (Fig. 4).
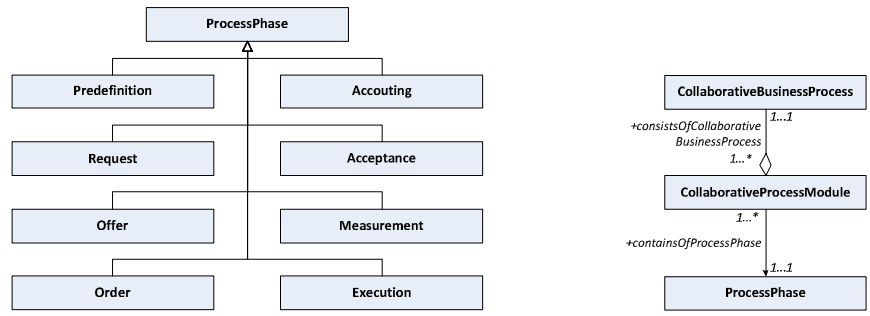

Fig. 4. Extensions of Process Phase and CollaborativeBusinessProcess

\section{$5 \quad$ Meta-model Instance of Data Model Extension}

The extended meta-model represents relevant entities and dependencies of business processes and information flows for industrial service procurement in a structured manner. The meta-model requirements have been defined and evaluated in several use cases $[10,11]$. Based on the meta-model, existing e-business standards can be extended and new standards can be developed in order to fulfill the logic dependencies (related to service-dominant-logic [19]). Meta-model instances define data objects for information systems. Generated meta-model instances provide standardized semantics 
of descriptions with common understanding to improve the information exchange in service procurement collaborations. So far standardized semantics are not available due to a variety of heterogeneous data formats. The given example is related to the service object order referencing service descriptions agreed between buyer and supplier in a respective frame contract. Order is a document type and represents a typical model instance of the data model perspective. In Fig. 5, the meta-model instance of an order document and also an example of a runtime data object order is shown. An order document type consists of a header and a body part. The header describes metadata like the ID of an order, the date, the ContractID reference and the ServicespecificationID reference. The header part includes information about the service requester and Service provider with their IDs and their addresses. The body part lists the industrial service description as service positions. As a runtime example, an UML object diagram of the relationship between a body element and three service description positions is shown.

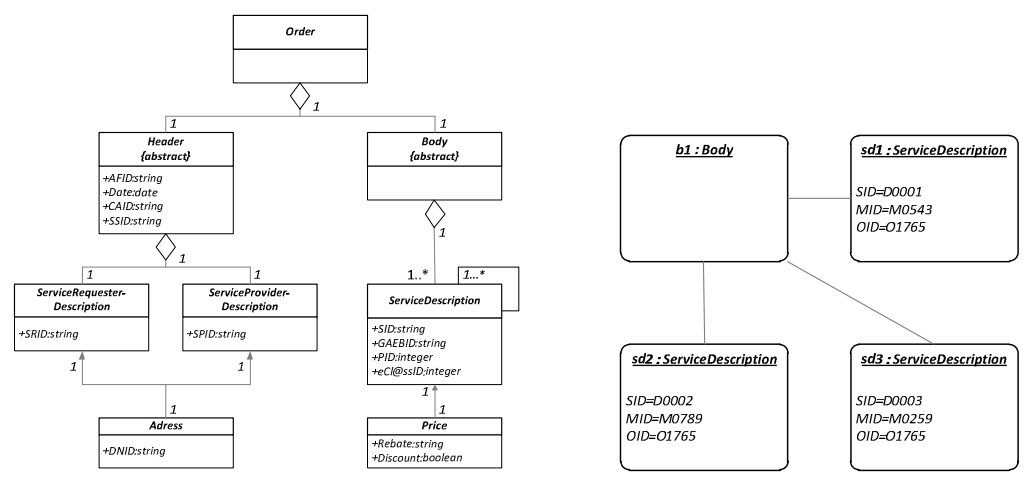

Fig. 5. Meta-model instance order of data model perspective and runtime example

\section{Conclusion and Outlook}

In this paper, a meta-model for business process compliance and data harmonization for service procurement collaborations was presented. It offers an integrated view on business processes and respective information flows. Furthermore a domain specific meta-model extension could be derived. The meta-models are information models supporting the design of process-aware information systems meeting the requirements of the flow of XML documents and the control flow of the underlying business process. Presented results have been applied and validated within the development of e-business standard concepts (based on the meta-model extension). Developed ebusiness standard concepts are currently being implemented in practice [11]. The meta-model was designed in conformity with hands-on requirements which could be elicited from real-life case-studies [10]. Next step foresees to elaborate further on the reference process model using a formal description language for modeling (such as Petri nets) and applying an integrated modeling approach enclosing various views and 
levels. Process and data model perspective were combined with the aim to design an abstract reference process model for service procurement. The meta-model extension also serves as reference system which captures best practices in industry. Furthermore it allows identifying and referencing relevant data objects by business partners using a shared framework.

\section{References}

1. Aurich, J.C., Schweitzer, E., Siener, M., Wolf, N.: Lebenszyklusorientierte Konfiguration investiver Produkt-Service-Systeme. Zeitschrift für wirtschaftlichen Fabrikbetrieb (ZWF) 102(12), 820-824 (2007)

2. Becker, J., Rosemann, M., Schütte, R.: Grundsätze ordnungsgemäßer Modellierung. In: Wirtschaftsinformatik, vol. 37(5), pp. 435-445. Vieweg+Teubner (1995)

3. Bullinger, H.-J., Fröschle H.-P., Brettreich-Teichmann, W.: Informations- und Kommunikationsinfrastruktur für innovative Unternehmen. Zeitschrift Führung und Organisation (4), 225-233 (1993)

4. Fettke, P., Loos, P.: Methoden zur Wiederverwendung von Referenzmodellen - Übersicht und Taxonomie. In: Becker, J., Knackstedt, R. (eds.) Referenzmodellierung - Methoden, Modelle, Erfahrungen. Arbeitsbericht No. 90 des Institut für Wirtschaftsinformatik, pp. 930. Westfälische Wilhelms-Universität Münster (2002)

5. Fließ, S.: Prozessorganisation in Dienstleistungsunternehmen, Stuttgart (2006)

6. Fowler, M.: Analysis Patterns: Reusable Objects Models. Addison-Wesley (1996)

7. Gebauer, H., Hildenbrand, K., Fleisch, E.: Servicestrategien für die Industrie. Harvard Business Manager 28(5), 47-55 (2006)

8. Meier, A., Stormer, H.: eBusiness \& eCommerce: Management der digitalen Wertschöpfungskette. Springer, Berlin (2008)

9. Herfurth, M., Weiß, P., Rudolf, C., Kern, R.: Reducing Complexity of Services. In: Böhmann, T., Leimeister, J.M. (eds.) Proceedings Teilkonferenz: Integration von Produkt und Dienstleistung: Hybride Wertschöpfung, Multikonferenz Wirtschaftsinformatik MKWI 2010 (2010)

10. Weiß, P., Herfurth, M., Schumacher, J.: Leverage Productivity Potentials in Serviceoriented Procurement Transactions: E-Standards in Service Procurement. In: Proceedings of RESER 2011 (2011)

11. Österle, H.: Geschäftsmodell des Informationszeitalters. In: Österle, H., Winter, R. (eds.) Business Engineering - Auf dem Weg zum Unternehmen des Informationszeitalters, Springer, Berlin (2003)

12. eBusInstand - Einsatz von Standards in der industriellenInstandhaltung, Research transfer project funded by Federal Ministry of Economics and Technology (2011),

http: / / www. ebusinstand. de

13. Rai, A., Sambamurthy, V.: Editorial Notes - The Growth of Interest in Service Management: Opportunities for Information Systems Scholars. Information Systems Research 17(4), 327-331 (2006)

14. Rinderle-Ma, S., Ly, L., Dadam, P.: Business Process Compliance. In: EMISA Forum, pp. 24-29 (2008)

15. Schuh, G., Schmidt, C.: Prozess. In: Schuh, G. (Hrsg.): Produktionsplanung und steuerung. Grundlagen, Gestaltung und Konzepte, pp. 108-194. Springer (2006) 
16. Smeltzer, L.R., Ogden, J.A.: Purchasing Professionals Perceived Differences between Purchasing materials and Purchasing Services. Journal of Supply Chain Management 38(19), 54-70 (2002)

17. Vargo, S.L., Lusch, R.F.: Evolving to a New Dominant Logic for Marketing. Journal of Marketing 68, 1-17 (2004)

18. Winkelmann, K.: Prospektive Bewertung der kooperativen Erbringung industrieller Dienstleistungen im Maschinenbau durch Simulation mit Petri-Netzen. Shaker Verlag (2007) 